\title{
In-diffusion and Out-diffusion of Oxygen from a Composite Containing Random Traps
}

\author{
I.V. Belova ${ }^{1, a}$ and G.E. Murch ${ }^{1, b}$ \\ ${ }^{1}$ Centre for Mass and Thermal Transport in Engineering Materials \\ School of Engineering \\ The University of Newcastle \\ Callaghan \\ NSW 2308 \\ Australia \\ a Irina.Belova@newcastle.edu.au, ${ }^{\text {b }}$ Graeme.Murch@newcastle.edu.au
}

Keywords: composites, segregation, diffusion, Lattice Monte Carlo

\begin{abstract}
In this paper, we investigate oxygen in-diffusion and out-diffusion with respect to a cermet composite where oxygen segregates at the interface between the metal matrix phase and the ceramic oxide phase. This phenomenological diffusion problem is treated by overlaying it with a fine-grained lattice that was addressed using a Lattice Monte Carlo method and a little-known exact expression for the lattice-based effective diffusivity in the presence of random traps. It is shown that there is very good agreement for the oxygen concentration depth profiles between the Monte Carlo results and the exact expression.
\end{abstract}

\section{Introduction}

During the initial synthesis and later in-service lifetime of a ceramic oxide-cermet, oxygen from the external surface diffuses through the metal matrix and segregates at the interfaces of the metal/ceramic oxide inclusions [1]. The presence of this oxygen leads to significant weakening of the bonding between the metal matrix and the ceramic oxide inclusions and subsequent serious deterioration of the mechanical properties of the cermet. This oxygen can generally be removed by annealing of the composite in vacuum at high temperature by an out-diffusion process that is essentially diffusion-limited evaporation [1]. Both processes of in-diffusion and out-diffusion are phenomenological mass diffusion problems that are amenable to being addressed using finitedifference and finite element methods. Recently, another method, termed the Lattice Monte Carlo (LMC) method, has been developed to address phenomenological mass as well as heat diffusion problems [2]. This method shows special promise as an alternative that is particularly well-suited for addressing diffusion problems that have complex geometries and/or which have singularities in the sources and sinks of diffusant.

The LMC method has been used to describe the detailed process of in-diffusion and segregation of oxygen at the interfaces of (square) $\mathrm{MgO}$ inclusions in an $\mathrm{Ag}$ matrix [3,4]. In this case, there was very good agreement of the local oxygen concentration profiles obtained from the LMC method with those obtained by the finite element method. In the present paper we take what might be regarded as a 'bigger picture' model of this phenomenological diffusion problem wherein the ceramic oxide inclusions are distributed randomly in the matrix and can be represented as featureless traps for oxygen within the matrix. In this paper, we investigate the effective diffusivity of oxygen in this model and deduce concentration profiles for both in-diffusion and out-diffusion using the LMC method. We also make contact with an exact lattice-based expression for the effective long-time limit diffusivity for a diffusant in the presence of random traps. 


\section{The Model}

In keeping with the spirit of the LMC method [2] a fine-grained lattice is constructed to overlay the phenomenological diffusion problem. For convenience, in this problem we use a 2D lattice. Each inclusion is represented as a single trap situated at a given site of this lattice. Accordingly, the mesh size of the lattice then determines the apparent size of the inclusions. The trapping sites are distributed randomly. In adopting such a model we are assuming in effect that the oxygen mobility along the interfaces of the metal/ceramic oxide inclusions is very rapid compared with diffusion in the matrix. Thus oxygen can exit (and enter) a trap with exactly the same probability in all four directions. This seems to be quite a reasonable simplifying assumption. We define $D_{1}$ as the oxygen diffusion coefficient in the matrix and $D_{2}$ as the diffusivity of oxygen associated with the interface i.e. with the trap itself. The diffusivities $D_{\mathrm{i}}$ are related to the jump frequencies $\Gamma_{\mathrm{i}}$ by the lattice random-walk expression in two dimensions:

$$
D_{\mathrm{i}}=\Gamma_{\mathrm{i}} a^{2} / 4
$$

where $a$ is the lattice mesh size and the factor 4 comes from the lattice being two dimensional. For a three dimensional lattice this factor would be 6 . The segregation factor $s$ is defined in the usual Henry's Law form (but a site blocking-type expression can also be used in principle [5]):

$$
s=\frac{\psi_{O}^{2}}{\psi_{O}^{1}}
$$

where $\psi_{O}^{2}$ and $\psi_{O}^{1}$ are the equilibrium concentrations of the diffusant oxygen at the traps and in the matrix respectively. The segregation factor is also given by:

$$
S=\Gamma_{12} / \Gamma_{21}
$$

where $\Gamma_{12}$ and $\Gamma_{21}$ are the jump frequencies into and out of a trap from the matrix. For convenience, in the absence of more detailed information, we set $\Gamma_{12}$ equal to $\Gamma_{1}$ and $\Gamma_{21}$ equal to $\Gamma_{2}$. Thus we have a simple two-frequency trapping model. For this trapping model, there exists a little-known exact lattice-based expression for the effective long-time diffusivity in the presence of random traps [6]. In the present notation, the expression is:

$$
\frac{D_{e f f}}{D_{1}}=\frac{\Gamma_{2}}{(1-g) \Gamma_{2}+g \Gamma_{1}}, \quad \frac{D_{e f f}}{D_{2}}=\frac{\Gamma_{1}}{(1-g) \Gamma_{2}+g \Gamma_{1}}
$$

where $g$ is the volume (area) fraction of the inclusions and $D_{\text {eff }}$ is the effective diffusivity of the composite. In effect, with respect to the lattice, the area of a given trap is $a^{2}$.

\section{Results and Discussion}

The expression Eq. 4 for the effective diffusivity was first verified by making use of the EinsteinSmoluchowski Equation:

$$
D_{\text {eff }}=<R^{2}>/ 4 t
$$

where $R$ is the displacement of a given particle in time $t$ and the Dirac brackets $\langle>$ indicates an average over a large number of particles. Eq. 5 is readily realized in an equilibrium simulation wherein completely non-interacting particles are permitted to diffuse in a lattice containing random traps for a fixed number of jump attempts per particle (proportional to time) [2]. We explored the 
situation where $D_{1}$ is greater than $D_{2}$ which is of course appropriate for the diffusion in the cer-met and, for completeness, we also investigated the converse situation. A range of values of the segregation factor was investigated. As can be seen in Fig. 1, the LMC results reveal that the latticebased expression [6] for the effective long-time limit diffusivity in the presence of random traps is indeed correct.

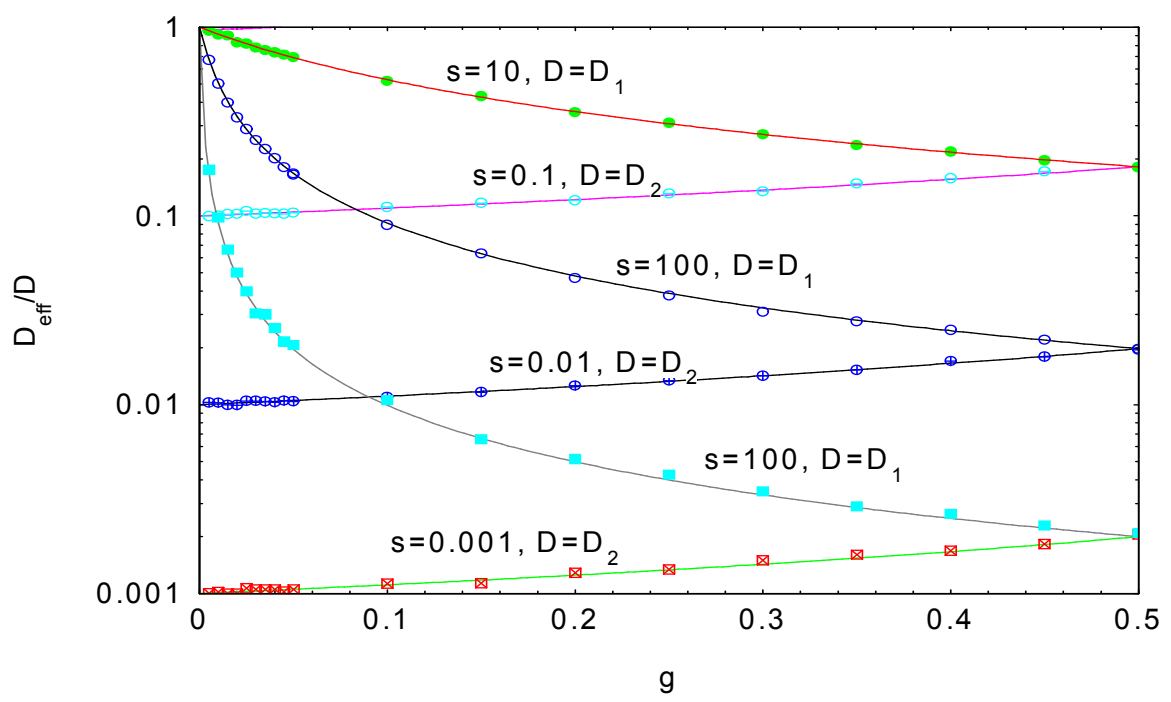

Figure 1. Calculated (symbols, Monte Carlo simulations) and theoretical values (solid lines, Eq. 4) for the ratios of the effective diffusivity $D_{\text {eff }}$ to $D$ as a function of $g$ - fraction of the random inclusions at several values of $\mathrm{s}$.

Next, we determined the oxygen concentration depth profiles for both in-diffusion and out-diffusion (with the constant source/sink boundary condition) of oxygen in the presence of random traps for reasonable values of the ratio of the matrix and trap diffusivities and segregation factor. For simulating the phenomenological process of in-diffusion from a constant surface source of oxygen into a solid we followed the procedure described in detail in [2,3]. For simulating the process of out-diffusion the procedure described in [2] was followed in general terms. We started with an equilibrium concentration of oxygen in the composite according to a specified value of the segregation factor. At diffusion times greater than zero the surface concentration of oxygen $\Psi_{0}$ was set equal to zero. This ensured a net flux of oxygen out from the model composite. In both cases of in-diffusion and out-diffusion at relatively short diffusion times the finite lattice sample can be considered infinite in the sense that the standard solutions of the Diffusion Equation can be employed. These solutions are:

In-diffusion

$$
\psi_{O}^{e f f}(x, t)=\psi_{O}^{e f f}(0, t) \operatorname{erfc}\left(\frac{x}{\sqrt{4 D_{e f f} t}}\right)
$$

Out-diffusion

$$
\psi_{O}^{e f f}(x, t)=\psi_{O}^{e f f}(x, 0) \operatorname{erf}\left(\frac{x}{\sqrt{4 D_{e f f} t}}\right)
$$

Figs. 2, 3 show results for the oxygen concentration profiles at different in-diffusion times and for $s$ $=10$ and 100 respectively. It is seen that there is generally very good agreement between the LMC 
results (symbols) and the expression (Eq. 4) for the exact long-time limit effective diffusivity in the presence of traps and Eq. 6 (shown by lines). At short diffusion times, there are small deviations because the long-time limit effective diffusivity is then not appropriate (these deviations are exacerbated at larger degrees of trapping i.e. larger segregation factors).

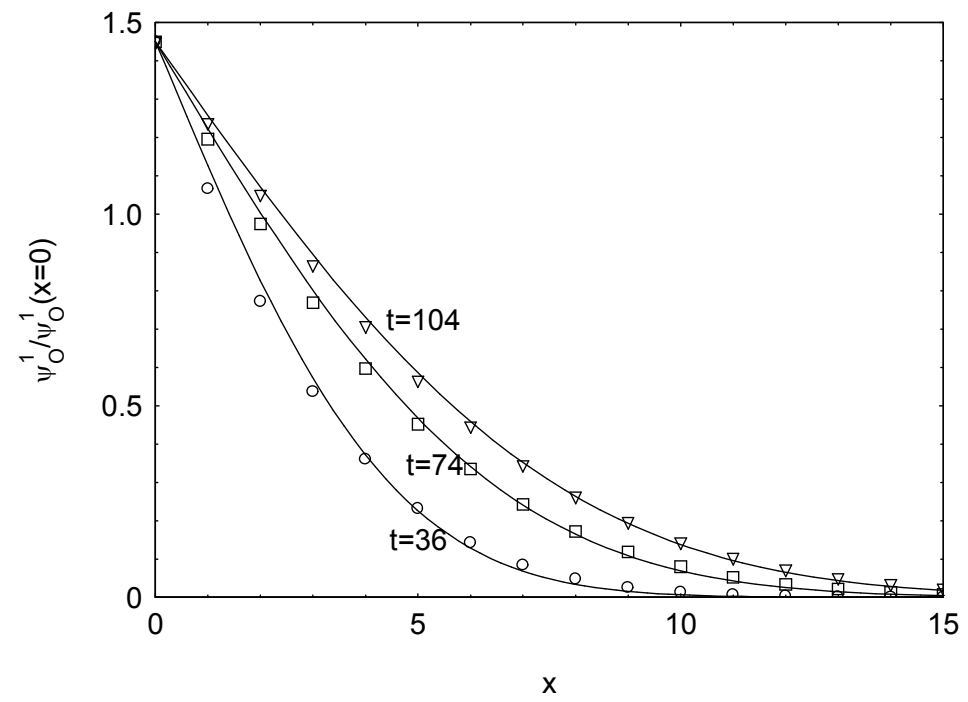

Figure 2. Oxygen concentration profiles at different in-diffusion times and for $\mathrm{s}=10$.

Fig. 4 shows results for the oxygen concentration profiles at different out-diffusion times for $\mathrm{s}=$ 100 and a constant initial composition. Again it is seen that there is generally very good agreement between the LMC results (symbols) and the expression (Eq. 4) for the exact long-time limit effective diffusivity in the presence of traps and Eq. 7 (shown by lines). Again, there are small deviations at short diffusion times for the same reasons as given above.

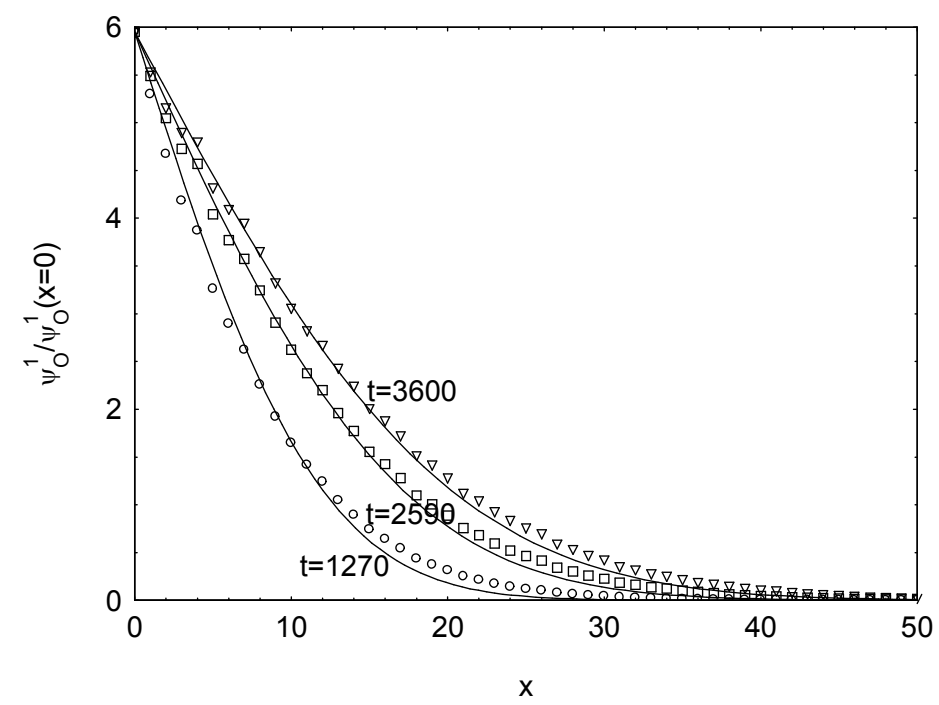

Figure 3. Oxygen concentration profiles at different in-diffusion times and for $\mathrm{s}=100$. 


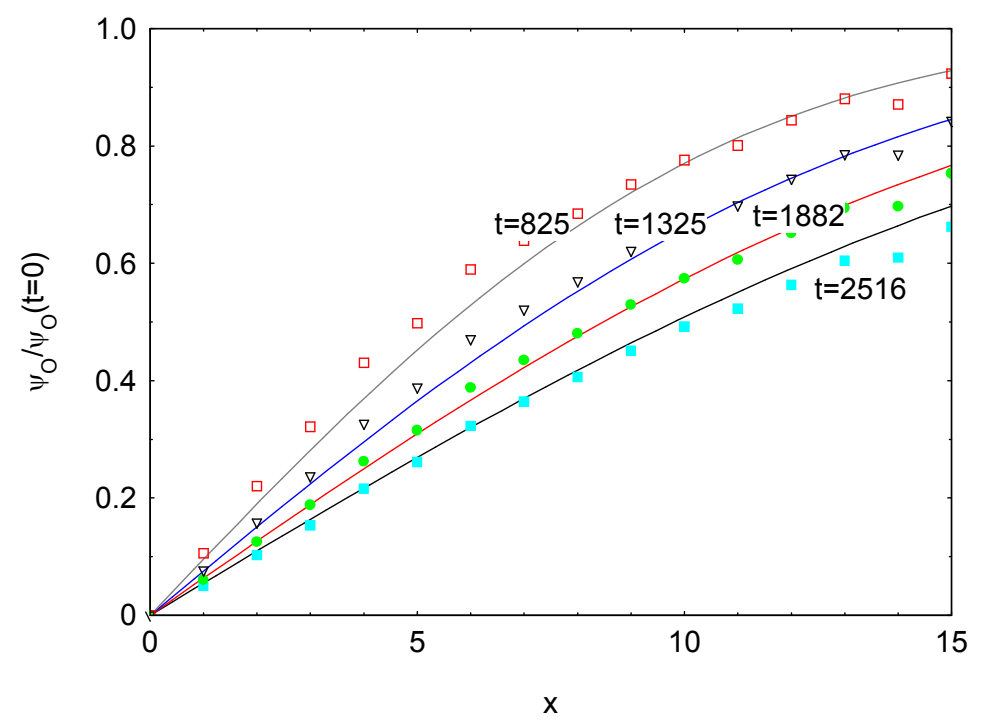

Figure 4. Oxygen concentration profiles at different out-diffusion times and for $\mathrm{s}=100$.

\section{Summary}

In this paper, we investigate oxygen in-diffusion and out-diffusion with respect to a cer-met composite where oxygen segregates at the interface between the metal matrix phase and the ceramic oxide phase. This phenomenological diffusion problem is treated by overlaying it with a finegrained lattice and making use of a Lattice Monte Carlo method. A little-known exact expression for the lattice-based effective diffusivity in the presence of random traps was also employed. It was shown that there is very good agreement for the oxygen concentration depth profiles between the Monte Carlo results and the exact expression.

\section{Acknowledgments}

We wish to thank the Australian Research Council for its support of this work.

\section{References}

[1] E. Pippel, J. Woltersdorf, J. Genger and R. Kirchheim, Acta Mater., Vol. 48 (2000), p. 2571.

[2] I.V. Belova and G.E. Murch: Solid State Phenomena, Vol. 129 (2007), p. 1.

[3] I V. Belova, N. Muthubandara, G E. Murch, M Stasiek, A Oechsner: Solid State Phenomena, Vol. 129 (2007) p.111.

[4] I.V. Belova, G.E. Murch, A. Oechsner, Defect and Diffusion Forum, Vol. 266, (2007) p. 29.

[5] I. Kaur, Y. Mishin and W. Gust, Fundamentals of Grain and Interphase Boundary Diffusion, Wiley, Chichester, 1995.

[6] J.W. Haus and K.W. Kehr, Physics Reports, Vol. 150 (1987), p. 263 